Onkologie 1988;11:299-302

\title{
Inhalt, Vol. 14, 1988
}

Impressum 2

Editorial 4

Kurrle, E. (Ulm) et al.

Induktions- und intensivierte Konsolidationstherapie der

akuten myeloischen Leukämie des Erwachsenen 5

Hiddemann, W. (Münster) et al.

Hochdosiertes Cytosin-Arabinosid und Mitoxantron: Erste Ergebnisse einer Pilotstudie über sequentielle Applikation (S-HAM) mit Hinweis auf eine hone anti-leukämische Aktivität bei refraktären akuten Leukämien 10

Leserzuschrift 12

Jehn, U. (München) et al.

Intensive Erhaltungstherapie bei akuter myeloischer Leukämie (AML): Ergebnisse eines einzelnen Instituts

im Rahmen einer randomisierten Multicenter-Studie ... 13

Hoelzer, D. (Frankfurt) et al.

Intensivierte Konsolidierungstherapie für ALL-Hoch-

risikopatienten 18

Hehlmann, R. (München) et al.

Chemotherapie der CML. Stand der deutschen Multi

center-Studie Busulfan versus Hydroxyurea versus Inter-

feron-alpha, Oktober $1987 \quad 25$

Siegert, W. (Berlin) et al.

Multizentrische prospektive risikoadaptierte Studie zur

Therapie von Non-Hodgkin-Lymphomen hoher Malignität. Einsatz von COP-BLA/IMVP-16 und randomisierte

adjuvante Strahlentherapie - Studienkonzept und vorläufige Ergebnisse 30

Ho, A. D. (Heidelberg) et al.

Desoxycoformycin bei der Behandlung refraktärer

Lymphome 35

Informationen für die Klinik 38

Peest, D. (Hannover) et al.

Induktions- und Erhaltungstherapie beim multiplen

Myelom. Ergebnisse einer multizentrischen Studie .... 39

Pralle, H. (Gießen) et al.

Interferon $\alpha$ für die Therapie der HCL. Ergebnisse von

drei prospektiven multizentrischen Studien in der Bun-

desrepublik 44

Pfreundschuh, M. (Köln) et al. 
Therapie der Hodgkin-Lymphome. Ergebnisse der Deut schen Hodgkin-Studiengruppe 48

Ganser, A. (Frankfurt) et al.

Der therapeutische Effekt von rhGM-CSF bei Patienten

mit myelodysplastischen Syndromen - eine Phase-I/

M-Studie 53

Beelen, D. W. (Essen) et al.

Behandlung der akuten Graft-versus-Host-Reaktion nach allogener Knochenmarktransplantation mit einem gegen den T-Zell-Rezeptor gerichteten monoklonalen Antikör-per (BMA031). Erste Ergebnisse einer Phase-I/II-Studie 56

Hausmaninger, H. (Salzburg) et al.

Vindesin-Mitoxantron (VM) versus Vindesin-4'-Epi-

doxorubicin (VE) bei der Behandlung des metastasieren-

den Mammakarzinoms: eine randomisierte prospektive

Studie 59

Mitrou, P. S. (Frankfurt) et al.

Die Behandlung des fortgeschrittenen und progredienten

epidemischen Kaposi-Sarkoms (EKS): Eine Phase-II-

Studie 61

Szuwart, U.; König, H.-J.; Bennefeld, H.; Weritz, Ch.;

Kleinhans, G. (Münster)

Klinik der hypophysären Metastasierungen 66

Informationen für die Klinik 70

Holtkamp, W.; Wuttke, W.; Nagel, G. A.;

Blossey, H. Ch. (Göttingen)

Vergleichende Untersuchungen zum Prolaktin-, Östrogen-, Gestagen- und Androgenrezeptorgehalt mensch-

licher Mammakarzinome 71

300

Jahres-Inhalt 1988

Meyer-Lindau, F.; Pfister, E.; Gyr, N.; Obrecht, J. P.

(Basel)

Randomisierte Doppelblindstudie über die analgetische

Wirkung von Caerulein und Morphin bei chronischen

Tumorschmerzen 77

Wowra, B.; Mentrup, E.; Zeller, W. J.; Strieker, H.;

Sturm, V. (Heidelberg)

CT-Kinetik intratumoraler Liposomendepots 81

Holtkamp, W.; Wuttke, W.; Nagel, G. A.; Michel, U.; Rauschecker, H. (Göttingen)

Pathophysiologie der Hyperprolaktinämie beim Mamma-

karzinom 86

Buchbesprechungen 104

Kurzmitteilung

Görg, C; Görg, K.; Pflüger, K.-H.; Havemann, K.;

Glanz, H.; Kleinsasser, O. (Marburg) 
Therapie des adenoidzystischen Karzinoms mit Cisplatin,

Etoposid und Vindesin 106

Obrist, R.; Battegay, M.; Obrecht, J. P.

(Basel/Schweiz)

Mediastinale Hodgkinsche Krankheit. - Eine retrospek-

tive Analyse 112

Henß, H.; Fiebig, H. H.; Schildge, J.; Arnold, H.;

Hasse, J. (Freiburg)

Phase-II-Studie der Kombination von Cisplatin und

Adriamycin bei fortgeschrittenen Pleuramesotheliomen . 118

Holtkamp, W.; Nagel, G. A. (Göttingen)

Bromokriptin beim chemotherapieresistenten, metasta-

sierten Mammakarzinom. - Ergebnisse der AlO-Studie

GÖ-MC-BROMO 2/82 121

Sonderbände 128

Klima, A.; Bettinger, R. (Frankfurt/M.)

Pathohistologische Serienschnittuntersuchungen bei

Patienten mit kompletter Tumorremission nach durch-

geführter zytostatischer Induktionstherapie 129

Informationen für die Klinik 138

Buchbesprechungen 140

Herrmann, G.; Hübner, K. (Frankfurt/M.)

Fibrosierende Alveolitis nach kombinierter Strahlen- und

Chemotherapie. - Ein Fallbericht 142

Kurzmitteilung

Queißer, W. (Mannheim); Herrmann, F.; Lindemann, A.

(Mainz); Anger, B. (Ulm); Hiddemann, W. (Münster);

Krey, U. (Kiel)

Therapie der Blast-Phase der chronischen granulozyti-

schen Leukämie mit Mithramycin und Hydroxykarbamid 145

Editorial 150

Giese, M.; Kirchner, H. (Heidelberg)

Interferone und ihre Wirkungen 151

Schwabe, M.; Wagner, L.; Prischl, F,; Pinter, G.; Schwarzmeier, J. D. (Wien/Österreich) Zum

Wirkungsmechanismus von Interferon- $\alpha 2$ bei Haar-zellen und lymphoblastoiden Zellinien: Die

Bedeutung von Typ-I-Interferon-Rezeptoren und RNS-Synthese . . . 155

Brünig, K.; Fülle, H. H.; Dahmen, E.;

Hellriegel, K.-P. (Berlin)

Therapie der Haarzell-Leukämie mit Interferon

Stadler, R.; Bratzke, B.; Mayer da Suva, A.;

Orfanos, C. E. (Berlin)

Therapie des AIDS-induzierten Kaposi-Sarkoms und des

malignen Melanoms mit Interferonen 166

Fülle, H. H.; Dahmen, E.; Hellriegel, K.-P. (Berlin)

Therapie der chronischen myeloischen Leukämie mit

Interferon

177 
Informationen für die Klinik 184

Otto, U.; Schneider, A.; Denkhaus, H.;

Conrad, S. (Hamburg)

Die Behandlung des metastasierenden Nierenkarzinoms

mit rekombinantem $\alpha$-2 oder $\gamma$-Interferon. - Ergebnisse

zweier klinischer Phase-II-bzw. -IП-Studien 185

Jahres-Inhalt 1988

301

Ludwíg, H.; Scheithauer, W.; Kiihrer, I.; Kuzmits, R.;

Gísslinger, G.; Linkesch, W.; Zielinski, Ch.;

Fritz, E. (Wien/Österreich)

Die Interferontherapie beim multiplen Myelom

Laudatio 200

Hölzel, D.; Sauer, H.; de Waal, J- C. (München)

Tumornachsorgeschemata: Wissensinhalt - Anwendung -

Optimierung 202

Schenck, U.; Jutting, U.; Eiermann, W. (München)

Hormonrezeptorbestimmung beim Mammakarzinom mit

der Dextran-Coated-Charcoal-Methode und mit mono-

klonalen Antikörpern: Korrelation mit einem zytomor-

phologischen Grading 211

Schillinger, H.; Traeder, R.; Klosa, W.; Pohl, J. (Freiburg)

Nachweis von Lebermetastasen gynäkologischer Mali-

gnome durch Sonographie, Szintigraphie, Computer-

tomographie und Leberenzyme 216

Informationen für die Klinik 221

Wolf, M.; Havemann, K.; Stalleicken, D.; Gropp, C; Maasberg, M.; Braun, C. (Marburg); Hans, K. (Oberhausen); von Bülzingslöwen, F. (Donaustaufen); Klasen, H. (Oldenburg); Becker, H.; Häßler, R. (Fulda); Schroeder, M. (Duisburg); Hirschmann, H.; Gerdes, H. (Kassel); Firuska, E. (Gerlingen); Mende, S. (Ravens-burg); Pieritz, H. G. (Mayen); Holle, R. (Heidelberg) Ergebnisse zweier multizentrischer Therapiestudien beim inoperablen nichtkleinzelligen Bronchialkarzinom .... 222

Sonderbände 231

Schaefer, H,-J.; Ollenschläger, G.; Madder, B. (Köln)

Orale Polychemotherapie bei weit fortgeschrittenen

Rezidiven des Morbus Hodgkin 233

Kolaric, K.; Zupanc, D. (Zagreb/Jugoslawien);

Stahl, K. W.; Hinz, J.; Kempf, S. R.; Ivankovic, S.

(Heidelberg)

Verhinderung von Extravasatnekrosen als Komplikation nach intravenöser Zytostatikatherapie. - Ergebnisse ei-

ner offenen Pilotstudie 238

Paasen, A.; Stimmel, A.; Neumann, H. A. (Bochum)

Übertragung von INMEDD-Dateien auf einen Perso-

nalcomputer. Ein neues Konzept zur Flexibilisierung des 


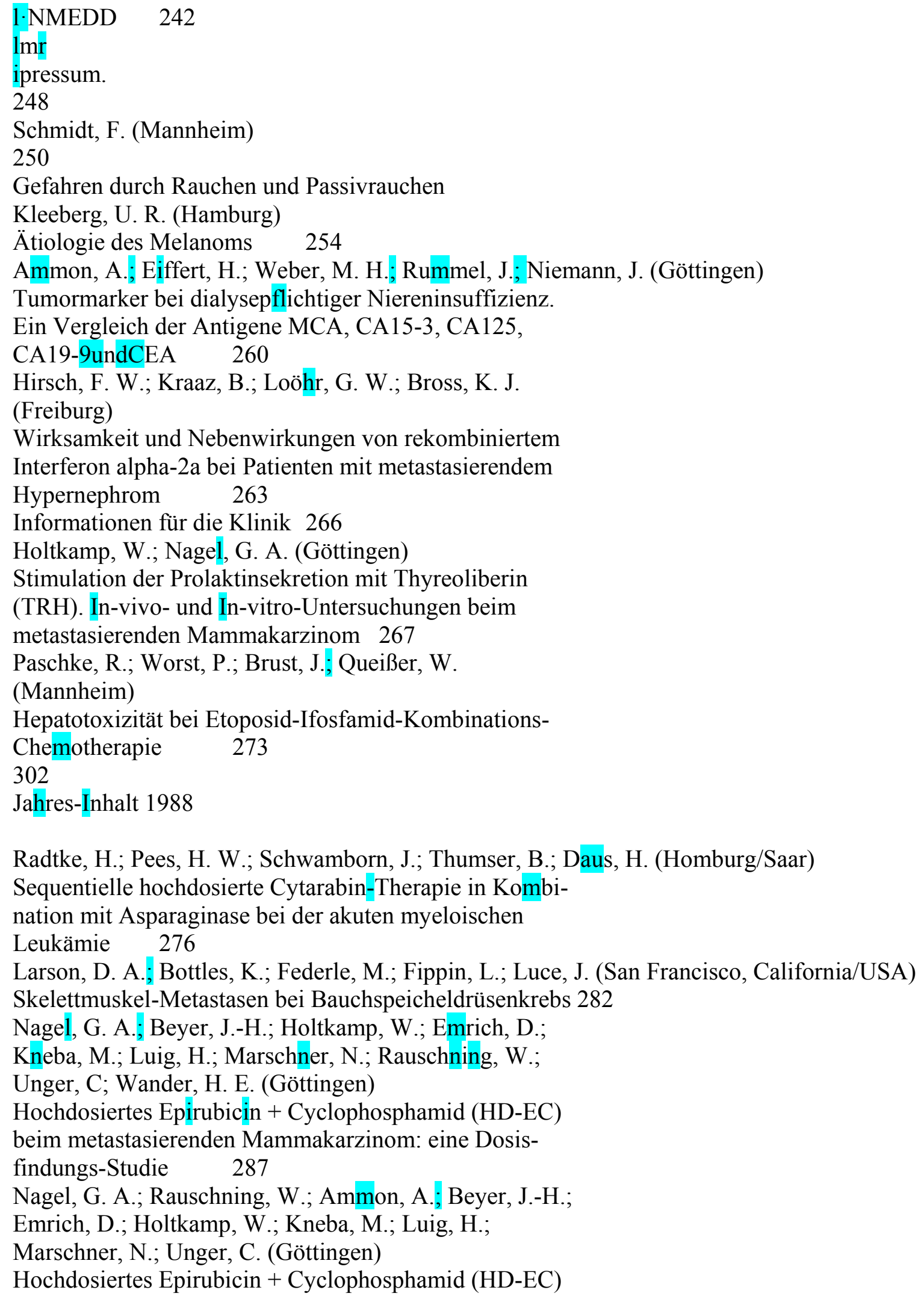


beim metastasierenden Mammakarzinom: Daten einer

Phase-II-Studie

289

Kreis, W.; Budman, D. R.; Delli Bovi, P.; Vinciguerra, V. (Manhasset, N.Y./USA)

Rascher Anstieg von Serum-Testosteron nach Beendi-

gung einer Langzeitbehandlung beim Prostatakarzinom

mit einem LHRH-Agonist 292

Sonderbände 293

Kurzmitteilung 295

Unger, C.; Eíbl, H.; Breíser, A.; von Hey den, H. W.; Nagel, G. A. (Göttingen); Engel, J.;

Hilgard, P.; Sindermann, H.; Peukert, M. (Bielefeld) Hexadecylphosphocholin (D18506) bei der

topischen Be-handlung von Hautmetastasen: Eine Phase-I-Studie . . 295

Autorenverzeichnis $1988 \quad 297$

Jahres-Inhalt $1988 \quad 299$

Supplement 1/Oktober 1988

Ovarialkarzinom

Empfehlungen zur Diagnostik, Therapie und Nachsorge

(Projektgruppe «Ovarialkarzinom» im Tumorzentrum

München) 1-32

Supplement 2/Dezember 1988

Schaefer, U. W. (Essen)

Knochenmarktransplantationen am Universitätsklinikum

Essen 3

Zschaber, R.; Koschel, G.; Kaukel, E.; Heilmann, H. P.;

Hossfeld, D. K. (Hamburg), Gatzemeier, U. (Großhans-

dorf)

Phase-III-Therapiestudie bei Patienten mit kleinzelligem

Bronchialkarzinom 9

Migeod, F.; Gerlach, D.; Kress, M.; Hoffmann, W.;

Farroukh, R.; Seeber, S. (Leverkusen)

Sequentielle Behandlung des progredient metastasieren

den kolorektalen Karzinoms mit 5-Fluorouracil/Folin-

säure, Dipyramidol und Mitomycin C

14

Firusian, N. (Recklinghausen)

Chemotherapie-Lasertherapie der fortgeschrittenen Tu-

moren des oberen Gastrointestinaltraktes 22

Niederle, N.; Eberhardt, W.; Hirsche, H. (Essen)

Prätherapeutische prognostische Faktoren beim kleinzel-

ligen Bronchialkarzinom 33

Sonderbände/Special Editions

39

Kloke, O.; May, D.; Wandl, U.; Niederle, N·. (Essen)

Zur Dosierung von Interferon alpha in der Induktions-

und Erhaltungstherapie der Haarzellenleukämie

Drings, P.; Becker, H.; Bülzebruck, H.; Djawid, N.;

Ruchalla, E.; Stiefel, P.; Tessen, H. W. (Heidelberg/

Rohrbach)

Die Chemotherapie des nicht-kleinzelligen Bronchialkar- 
zinoms mit Ifosfamid in Kombination mit Cisplatin, Etoposid oder Vindesin 47 